Harvard Kennedy School Misinformation Review ${ }^{1}$

May 2021, Volume 2, Issue 3

Creative Commons Attribution 4.0 International (CC BY 4.0)

Reprints and permissions: misinforeview@hks.harvard.edu

DOI: https://doi.org/10.37016/mr-2020-70

Website: misinforeview.hks.harvard.edu

\title{
Where's the fake news at? European news consumers' perceptions of misinformation across information sources and topics
}

This study indicates that news users across ten different European countries are quite concerned about misinformation in their information environment. Respondents are most likely to associate politicians, corporations, and foreign actors with misinformation. They perceive misinformation to be most common for topics like immigration, the economy, and the environment. This offers support for the increasingly more relative and politicized status of facts in people's credibility perceptions. Yet, differences across sources and issues are relatively modest, indicating that misinformation can be associated with many different information sources and topics.

Authors: Michael Hameleers (1), Anna Brosius (1), Claes H. de Vreese (1)

Affiliations: (1) Amsterdam School of Communication Research (ASCoR), University of Amsterdam, Netherlands

How to cite: Hameleers, M., Brosius, A., \& de Vreese, C. H. (2021). Where's the fake news at? European news consumers' perceptions of misinformation across information sources and topics. Harvard Kennedy School (HKS) Misinformation Review, 2(3).

Received: February 2nd 2021 . Accepted: April 2nd $2^{\text {nd }}$ 2021. Published: May 13 ${ }^{\text {th }}, 2021$.

\section{Research questions}

- RQ1: Which information sources do people associate with misinformation?

- RQ2: Which topics do people associate with misinformation?

- RQ3: Are topics that people find important more or less associated with misinformation?

\section{Essay summary}

- In a large-scale survey among nationally representative samples of citizens in ten European countries, 6,643 respondents indicated which sources they think disseminate false information, and which topics are most affected by misinformation.

- Our results indicate that misinformation is not just associated with highly polarized issues - such as climate change or immigration. Rather, perceptions of falsehoods are ubiquitous and assigned to all kinds of sources and topics.

\footnotetext{
${ }^{1}$ A publication of the Shorenstein Center on Media, Politics, and Public Policy at Harvard University, John F. Kennedy School of Government.
} 
- Politicians, corporations, and foreign actors are seen as the most likely disseminators of false information.

- People are most likely to associate misinformation with topics they find important.

- Although moderate levels of skepticism regarding information on important issues may be conducive to democratic ideals, strong distrust in all information may impede the acceptance of empirical evidence and expert knowledge.

- Interventions may be geared toward restoring trust in the motives of information sources through transparency about processes of verification and judging competing evidence.

\section{Implications}

The spread of inauthentic or erroneous information may be one of the most pressing issues facing digital information ecologies today (Bennett \& Livingston, 2018). In general, misinformation can be defined as untrue or inaccurate information (Wardle, 2017), or information that is deemed erroneous or untrue based on relevant expert knowledge (Vraga \& Bode, 2020). In this paper, we focus on misinformation as perceptions: Citizens' beliefs about the accuracy and truthfulness of depictions of reality. These misinformation beliefs can pertain to different sources, especially in today's high-choice information environment where many alternative and established sources of information compete for attention and legitimacy (Waisbord, 2018). In our study, we therefore focus on misinformation associated with information sources in general, which could, for example, relate to offline and online mainstream and alternative (news) media outlets, information from social media, or information from political elites and authorities (i.e., the WHO). As news users are likely to associate misinformation with a plethora of online and offline media sources (Nielsen et al., 2020), we decided to not restrict our endeavor to specific (digital) sources of (mis)information.

Based on evidence from a ten-country survey, we show that respondents are quite likely to believe that misinformation is a common issue that affects different sources and issues. Measured on a 7-point scale, the average score of misinformation perceptions was above the midpoint of the scale $(M=4.4, S D$ $=1.3$ ). The relatively high salience of misinformation beliefs corresponds to increasing factual relativism in society, and resonates with the omnipresence of attacks on the media's accuracy, media critique, and Fake News accusations in public and political discourse (Egelhofer \& Lecheler, 2019; Fawzi, 2019; Schulz et al., 2018).

\section{Sources of misinformation}

In our more detailed analyses (RQ1), we show that people with misinformation beliefs are most likely to associate false information with politicians, corporations, and foreign actors. They are less likely to associate the news media in general, the national government, and other citizens with misinformation. Citizens' assessment of false information corresponds to the sources that are allegedly most likely to spread false information (e.g., Bennett \& Livingston, 2018; Waisbord, 2018). Politicians and corporations can use ungated digital information channels (particularly social media) to directly communicate with their followers. As indicated in extant literature, the absence of gatekeepers in digital settings can offer a favorable opportunity structure for false information and counter-factual narratives (e.g., Waisbord, 2018). Traditional news media sources - i.e., online or offline mainstream news that impact public opinion, typically published by larger media corporations - are more bound by journalistic role perceptions, which include verification and truth-seeking (Borden \& Tew, 2007; Waisbord, 2018). However, the differences between sources are relatively small, and generally indicate that most citizens do not strongly differentiate between potential sources of misinformation. This aligns with the hybrid 
nature of disinformation campaigns (Kim et al., 2018; Zhang et al., 2021), and the participation of different actors and sources in the dissemination of misinformation (Starbird, 2019). Although mis- and disinformation may originate from counter-factual online communities and political actors, citizens and mainstream media amplify disinformation by sharing or commenting on false narratives. In addition, agents of disinformation employ 'troll armies' of regular (inauthentic) social media users to disseminate counter-factual narratives (e.g., Kim et al., 2018; Lukito, 2020). Our findings indicate that citizens are aware of these intertwined paths of misinformation's spread, which is a necessary aspect of correctly identifying misinformation. Connected to research on media literacy in an era of mis- and disinformation, our findings offer support for an optimistic perspective: Information literacy can help people to identify misinformation (Jones-Jang et al., 2019), and we show that the prevalence of misinformation from all kinds of sources in a 'participatory' network (Starbird, 2019) matches the perceptions of news users. Yet, we need more research that assesses the accuracy of misinformation beliefs across issues and topics.

\section{Misinformation perceptions across topics and issues}

Turning our attention to the topics that are most associated with falsehoods (RQ2), we see that people are likely to associate falsehoods with a number of issues. The topic of immigration is most likely to be seen as subject to false information. The economy and the environment also are seen as issues particularly surrounded by misinformation. These issues tend to be highly polarized in public discourse, which could also mean that more misinformation is disseminated on topics high on the political agenda in the European countries studied (Humprecht, 2018). This aligns with research on the partisan nature of disinformation campaigns (e.g., Bennett \& Livingston, 2018; Humprecht, 2018; Marwick \& Lewis, 2017; Pennycook \& Rand, 2018). This result has two alternative implications. First, news users may be more accuracy-motivated when processing information on polarizing issues known to be subject to falsehoods, such as immigration. Second, polarizing issues covered in the news may be subject to more doubt and distrust as news users are strongly divided on these issues. Right-wing populist voters, for example, tend to distrust the media and elites more. Such antagonisms in public opinion can explain the relatively high levels of perceived misinformation about immigration (Fawzi, 2019; Schulz et al., 2018).

\section{Who are most likely to classify information sources and issues as misinformation?}

These findings reveal that we have to take individual-level differences into account to explain differences in perceived misinformation (RQ3). News consumers with more pronounced populist attitudes perceiving a central divide between ordinary people and elites - are most likely to associate politicians with misinformation. This points to the counter-factual dimension of populist support: Populist voters may not only blame politicians for not representing the people, but also for distorting the truth or lying to the people (also see Fawzi, 2019; Schulz et al., 2018). This could also make them more receptive to counter-factual narratives in online communities (Waisbord, 2018).

Issue importance explains some differences in misinformation beliefs: The more people care about issues such as immigration, education, welfare, and the economy, the more they associate these issues with misinformation. Since these issues are considered important and are subject to societal and partisan debates, there are more alternative narratives and opinions, especially online, which may cultivate the perception that misinformation is widespread regarding these topics. If opposed issue-specific or ideological camps both believe that misinformation is spread on the topics they disagree on, this could result in truth polarization: Oppositional camps are not only separated based on their beliefs, but also disagree on the factual basis that forms the foundation for debate.

In the countries included in this study, immigration is the topic most associated with misinformation, which aligns with the centrality of this issue, especially in European countries that witnessed the refugee 
crisis and its politicization during the past two decades. We also see that misinformation perceptions resonate with different contextual opportunity structures across countries. In Greece and Spain, for example, recent economic recessions (i.e., the Euro crisis) correspond with the highest levels of misinformation perceptions on the economy. This supports the notion that misinformation beliefs are most pronounced for issues that are important on the individual and contextual level. As indicated by Humprecht (2018), the topics and issues surrounded by higher levels of misinformation strongly reflect national political agendas. In countries where immigration is a salient issue, for example, more misinformation on this issue is disseminated. In a similar vein, looking at perceptions of misinformation, we show that in countries where topics like immigration or the economy dominate political agendas, citizens are likely to also associate them with misinformation. News users thus seem to recognize the salience of misinformation on different topics across regions.

\section{Practical implications}

Based on our findings that show high levels of perceived misinformation across sources and issues, a practical recommendation for communicators and authorities is to restore trust and report as transparently as possible. Especially when it comes to polarizing issues such as immigration, it could be helpful to lay out the procedures of research, verification, and judging (competing) expert knowledge and evidence - corresponding to journalistic roles of investigating, disseminating, and fact-checking. This is also the case during acute crises, where high levels of uncertainty lead to inevitable errors in communication. Transparency may help to maintain healthy levels of skepticism, rather than promoting a cynical 'blanket rejection' of all information (see, e.g., Pinkleton et al., 2012). Acknowledging and appreciating the skepticism of news users, especially those who care strongly about the issue at hand, could also help refute accusations of deliberate distortions of reality. In light of the increasing attacks on the legitimacy of expert knowledge and empirical evidence (i.e., the omnipresence of Fake News labels), this is a key challenge for journalists in particular. These insights into procedures of (news) reporting can also be part of media literacy programs, that should help citizens to trust accurate information next to classifying untrue information as false.

\section{Findings}

Finding 1: Politicians are seen as most likely to spread misinformation.

To answer RQ1, asking which sources people associate with misinformation, we compare the mean scores on misinformation perceptions across sources. There are only small variations in the sources that citizens commonly associate with misinformation; however, there are statistically significant patterns (repeatedmeasures ANOVA; $F=93.0, p<.01$ ). Politicians are seen as disseminators of misinformation most commonly, followed by corporations, foreign countries, the news media, the national government, and other citizens. Figure 1 plots the differences. Based on Bonferroni-adjusted pairwise comparisons, the only pairs whose means are not significantly different are the government and citizens as well as the news media and foreign countries.

There are several interesting differences between countries (see Appendix for more details). Sources commonly associated with misinformation vary somewhat across countries; for example, politicians are seen as disseminators of misinformation particularly in Greece, Spain, Poland, France, and Hungary. In Germany, corporations are associated most with misinformation. In Sweden, Denmark, and the Netherlands, other citizens, foreign countries, and corporations are seen as culprits. Interestingly, the news media is not seen as the main disseminator of misinformation in any of the countries. When 
interpreting these differences, it is important to keep in mind that, while there are certain patterns, the differences are not large.

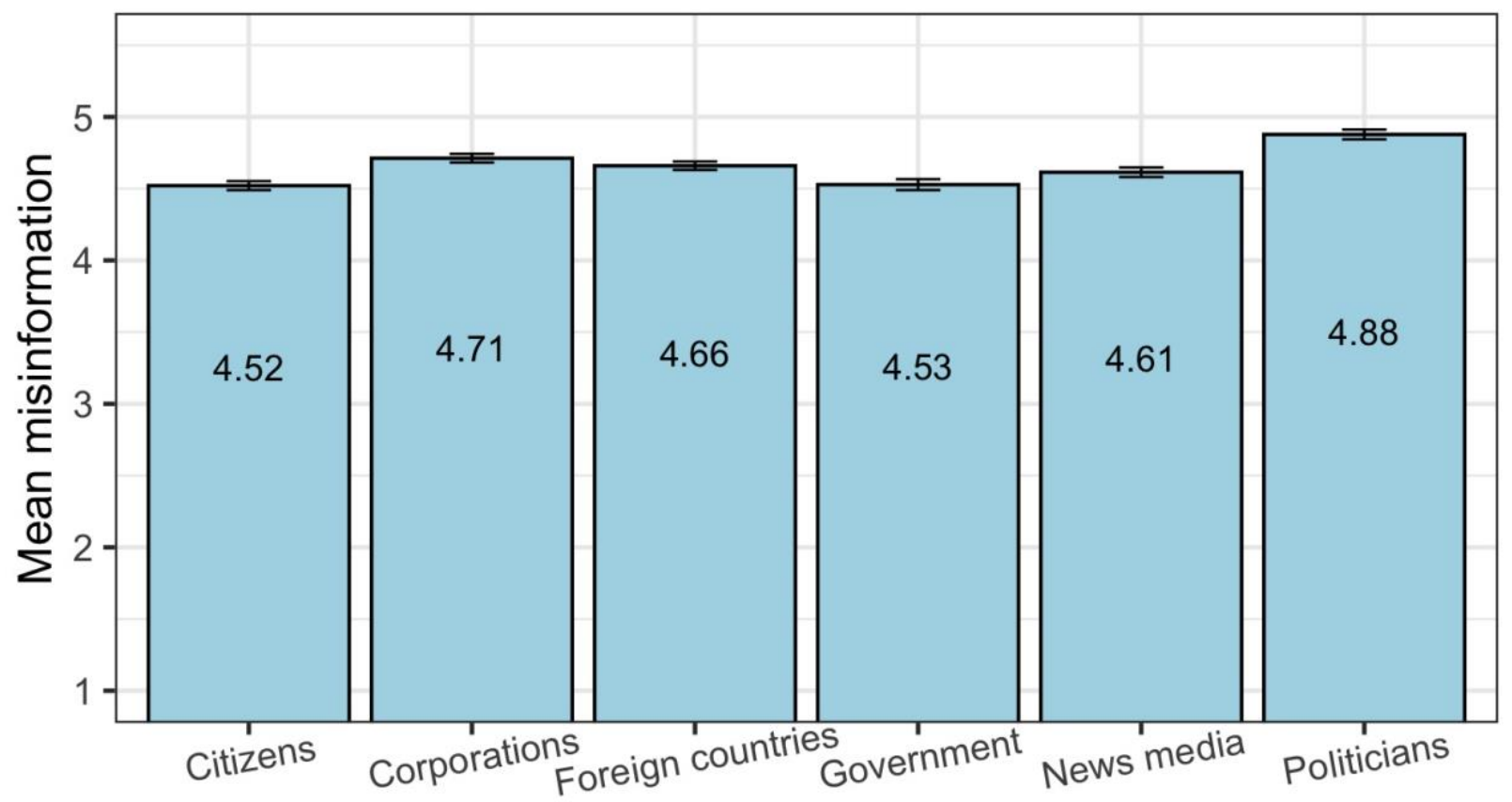

Sources

Figure 1. Mean misinformation perceptions for different sources. All perceptions are measured on 7-point scales (1= source never spreads misinformation, 7 = source spreads misinformation all the time).

Finding 2: Citizens think immigration is the topic most associated with misinformation.

RQ2 asked which topics people associate with misinformation (see Figure 2 for mean score comparisons). There are statistically significant differences between topics (repeated measures ANOVA; $F=362.6, p<$ .01). Immigration stands out as the topic most associated with misinformation, followed by the economy and the environment. After that follow international politics, terrorism, welfare, and the EU with relatively comparable levels of perceived misinformation. Education is least associated with misinformation. Based on Bonferroni-adjusted pairwise comparisons, international politics is not significantly different in its association with misinformation from topics of the environment, social welfare, and terrorism; the latter is not significantly different from the EU and social welfare. The topic of the EU is similarly associated with misinformation as the topic of social welfare. The same goes for the environment and the economy. All other differences are statistically significant, as also reflected in the larger effect sizes.

Regarding country comparisons (see Appendix for details), citizens perceive misinformation as most common within the topic of economy in Greece and Spain - countries that were more severely affected by the Euro crisis. Citizens also believe that the environment is one of the topics most impacted by misinformation in Germany, Denmark, Sweden, and the Netherlands. Immigration is a topic commonly associated with misinformation across all countries.

It is noteworthy that for all actors and topics, the degree of misinformation spreading associated with them is above the mid-point of the scale (4), meaning that citizens are on average quite concerned about the spread of misinformation by many actors across a variety of topics. 


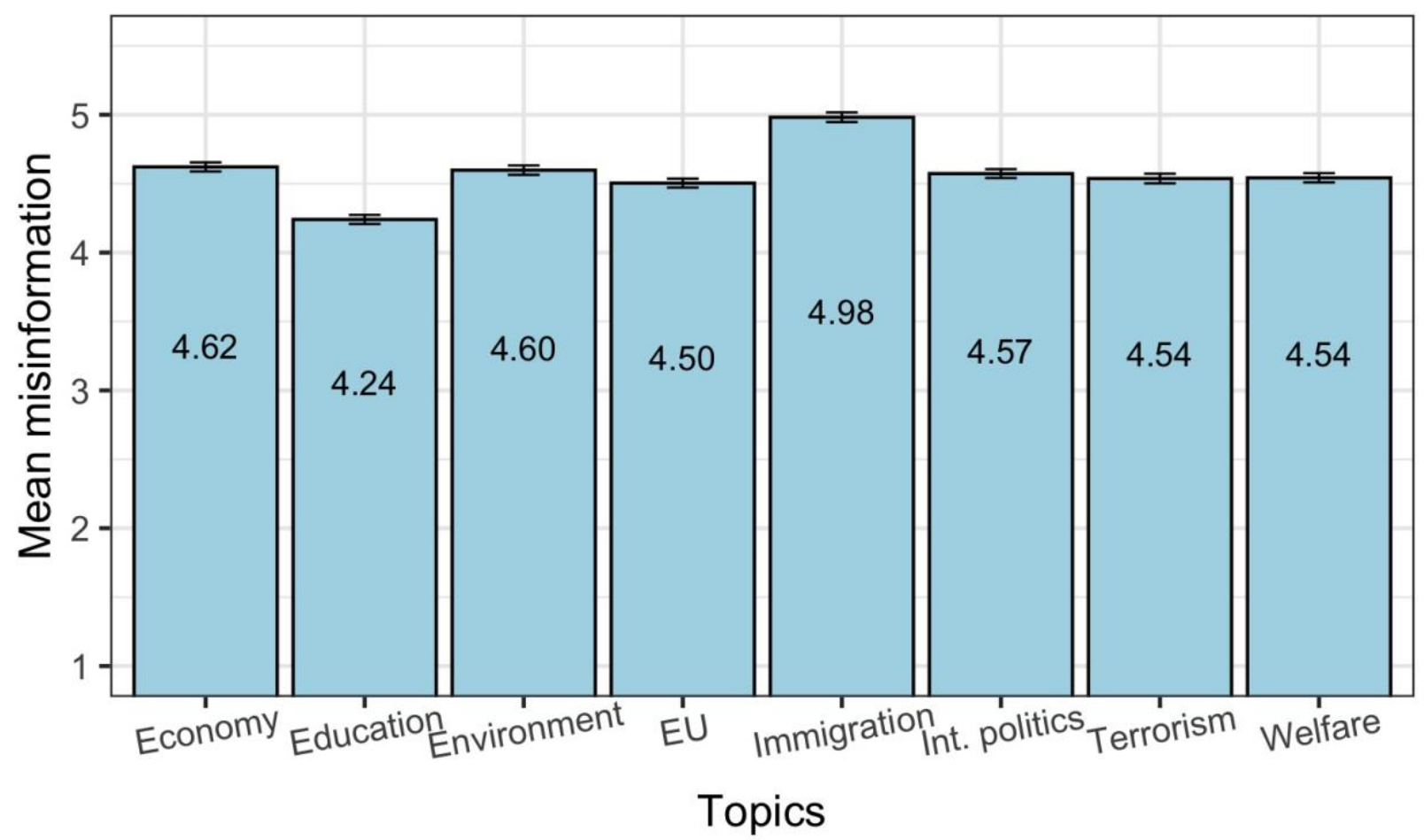

Figure 2. The topics associated most with misinformation. All perceptions are measured on 7-point scales (1= no inaccurate or dishonest information on this topic at all, 7 = very much inaccurate or dishonest information on the topic).

Finding 3: Citizens are more likely to associate topics that they find important with misinformation.

We finally asked whether topics that are deemed more important on the individual level are also more likely to be associated with misinformation (RQ3). Our data shows that associating politicians with misinformation is strongly related to anti-elite populist beliefs at $r=.62(p<0.01, \mathrm{df}=6,281)$. This means that people who perceive a central cleavage between the 'ordinary' people and the 'corrupt' elites are most likely to view politicians as sources of misinformation. This reveals an affinity between populist support and distrust in information spread by political elites. Those with higher levels of anti-immigration attitudes are also more likely to perceive higher levels of misinformation surrounding the topic of immigration ( $r=.14, p<.01, \mathrm{df}=6,641)$. These findings indicate that attitudes related to polarizing topics correspond to more pronounced misinformation beliefs.

Furthermore, we find that issue importance is often associated with misinformation perceptions. There are positive correlations between issue importance and misinformation perceptions for the issues of welfare $(r=.12, p<.01, \mathrm{df}=6,641)$, immigration $(r=.10, p<.01, \mathrm{df}=6,641)$, the economy $(r=.16, p<$ .01 , $\mathrm{df}=6,641)$, and education $(r=.16, p<.01 \mathrm{df}=6,641)$. However, this is not the case for all topics: There is no correlation between issue importance and misinformation perceptions regarding the environment ( $r=.01, p=.45, \mathrm{df}=6,641$ ) and only a weak negative correlation between misinformation beliefs regarding the EU and EU issue importance $(r=-.03, p=.03, \mathrm{df}=6,641)$.

\section{Methods}

We rely on a representative cross-country survey $(N=6,643)$ in which we included questions about the sources people associate with false content (RQ1); the issues people perceive to be subject to the dissemination of untruthful information (RQ2); and correlates of these misinformation beliefs (RQ3). 
Data collection was conducted by the international research company Kantar, using Computer Assisted Web Interviewing (CAWI). Members of mixed-source databases across ten countries voluntarily opted-in to participate in the survey. Recruitment into databases was based on various modes (i.e., e-mail invitations, telephone, face-to-face recruitment). Soft quotas were used to ensure a varied composition of demographics across all national samples. The data collection effort of this study was part of a larger seven-wave project on attitudes toward the European Union. Data used for this project were mostly collected in the final wave (July, 2019), with the exception of most sociodemographic variables and the variables measuring issue importance and anti-immigration attitudes. The numbers of respondents in the final wave per country are: Czech Republic $=733$, Germany $=518$, Denmark $=563$, Spain $=552$, France $=$ 776 , Greece $=494$, Hungary $=586$, the Netherlands $=1,067$, Poland $=857$, and Sweden $=497$. Respondents are 46.5 years old on average (SD $=15.7$ ); $50 \%$ are women. Age, gender, and region distributions approach national representativeness as closely as possible. Some respondents did not answer all questions; therefore, the $\mathrm{N}$ for some analyses is slightly lower due to missing values (minimum of 5,811 ).

To measure the sources associated with false information, we asked respondents "In your opinion, how often do the following sources of news spread inaccurate or false information?," referring to a) the news media, b) the national government, c) politicians [in country of respondent], d) corporations, e) ordinary citizens, and f) foreign countries. The sources were presented in randomized order. For each category, respondents could answer on a scale from 1 (never) to 7 (all the time). Respondents were able to indicate "I do not know."

To explore the topics associated with misinformation, we asked respondents "In your opinion, how much inaccurate or dishonest information is there in the media on the following issues?", referring to a) the European Union, b) the environment, c) social welfare, d) the economy, e) immigration, f) education, $\mathrm{g}$ ) international politics, and h) terrorism. The topics were presented in randomized order. The scale went from 1 (none at all) to 7 (very much).

We measured populist beliefs as the average agreement on a scale from 1 to 7 with three anti-elite statements (e.g., "politicians in government are corrupt"). Issue importance was measured on a 1 to 7 scale on which respondents could indicate how important specific issues were to them personally. Antiimmigration attitudes were measured as agreement with 3 anti-immigration statements and 2 reversed pro-immigration statements on a scale from 1 to 7; they were measured at different time points in the different countries.

Given the descriptive nature of the research questions, we conducted mostly descriptive analyses, focusing in particular on the sizes of mean differences. We tested the statistical significance of these mean differences using repeated measures ANOVA, given that all respondents were asked about all topics and sources. To determine group differences in detail, we conducted Bonferroni post-hoc tests, which indicated that most differences are also statistically significant.

\section{Bibliography}

Bennett, W. L., \& Livingston, S. (2018). The disinformation order: Disruptive communication and the decline of democratic institutions. European Journal of Communication, 33(2), 122-139. https://doi.org/10.1177/0267323118760317

Borden, S. L., \& Tew, C. (2007). The role of journalists and the performance of journalism: Ethical lessons from "fake" news (seriously). Journal of Mass Media Ethics, 22(4), 300-314. https://www.tandfonline.com/doi/abs/10.1080/08900520701583586

Egelhofer, J. L., \& Lecheler, S. (2019). Fake news as a two-dimensional phenomenon: A framework and research agenda. Annals of the International Communication Association, 43(2), 97-116. https://doi.org/10.1080/23808985.2019.1602782 
Fawzi, N. (2019). Untrustworthy news and the media as "Enemy of the People?" How a populist worldview shapes recipients' attitudes toward the media. The International Journal of Press/Politics, 24(2), 146-164. https://doi.org/10.1177/1940161218811981

Humprecht, E. (2018). Where 'fake news' flourishes: A comparison across four Western democracies. Information, Communication \& Society, 22(13), 1973-1988.

https://doi.org/10.1080/1369118X.2018.1474241

Jones-Jang, S. M., Mortensen, T., \& Liu, J. (2019). Does media literacy help identification of Fake News? Information literacy helps, but other literacies don't. American Behavioral Scientist, 65(2), 371388. https://doi.org/10.1177/0002764219869406

Kim, Y., Hsu, J., Neiman, D., Kou, C., Bankston, L., Kim, S. Y., ... Raskutt, G. (2018). The stealth media? Groups and targets behind divisive issue campaigns on Facebook. Political Communication, 35(4), 515-541. https://doi.org/10.1080/10584609.2018.1476425

Lukito, J. (2020). Coordinating a multi-platform disinformation campaign: Internet Research Agency activity on three U.S. social media platforms, 2015 to 2017. Political Communication, 37(2), 238255. https://doi.org/10.1080/10584609.2019.1661889

Marwick, A., \& Lewis, R. (2017, May 15). Media manipulation and disinformation online. Data and Society Research Institute. https://datasociety.net/output/media-manipulation-and-disinfoonline/

Nielsen, R. K., Fletcher, R., Newman, N., Brennen, J. S., \& Howard, P. N. (2020, April 15). Navigating the 'infodemic': How people in six countries access and rate news and information about coronavirus. Reuters Institute for the Study of Journalism. https://reutersinstitute.politics.ox.ac.uk/infodemic-how-people-six-countries-access-and-ratenews-and-information-about-coronavirus

Pennycook, G., \& Rand, D. G. (2018). Lazy, not biased: Susceptibility to partisan fake news is better explained by lack of reasoning than by motivated reasoning. Cognition, 188, 39-50. https://doi.org/10.1016/j.cognition.2018.06.011

Pinkleton, B. E., Austin, E. W., Zhou, Y., Willoughby, J. F., \& Reiser, M. (2012). Perceptions of news media, external efficacy, and public affairs apathy in political decision making and disaffection. Journalism \& Mass Communication Quarterly, 89(1), 23-39. https://doi.org/10.1177/1077699011428586

Schulz, A., Wirth, W., \& Müller, P. (2018). We are the people and you are fake news: A social identity approach to populist citizens' false consensus and hostile media perceptions. Communication Research, 47(2), 201-226. https://doi.org/10.1177/0093650218794854

Starbird, K. (2019). Disinformation's spread: Bots, trolls and all of us. Nature, 571(7766), 449.

https://doi.org/10.1038/d41586-019-02235-x

Vraga, E., \& Bode, B. (2020). Defining misinformation and understanding its bounded nature: Using expertise and evidence for describing misinformation. Political Communication, 37(1), 136-144. https://doi.org/10.1080/10584609.2020.1716500

Waisbord, S. (2018). Truth is what happens to news: On journalism, fake news, and post-truth. Journalism Studies, 19(13), 1866-1878. https://www.tandfonline.com/doi/abs/10.1080/1461670X.2018.1492881

Wardle, C. (2017). Fake news. It's complicated. First Draft. https://medium.com/1st-draft/fake-news-itscomplicated-d0f773766c79

Zhang, Y., Lukito, J., Su, M.-H., Suk, J., Xia, Y., Kim, S. J., Doroshenko, L., \& Wells, C. (2021). Assembling the networks and audiences of disinformation: How successful Russian IRA Twitter accounts built their followings, 2015-2017. Journal of Communication, 71(2), 305-331. https://doi.org/10.1093/joc/jqaa042 


\section{Funding}

The authors disclosed receipt of the following financial support for the research, authorship, and/or publication of this article: This research is funded by a grant from the European Research Council (ERC), grant number 647316.

\section{Competing interests}

The authors do not have any competing interests.

\section{Ethics}

The data collection was approved by the ethics committee for research involving personal data. Participants agreed on participation via voluntary opt-in and informed consent procedures. Participants could withdraw participation or fill out other/don't know categories for questions involving i.e., gender. All data was processed anonymously and on an aggregate level.

\section{Copyright}

This is an open access article distributed under the terms of the Creative Commons Attribution License, which permits unrestricted use, distribution, and reproduction in any medium, provided that the original author and source are properly credited.

\section{Data availability}

The dataset is available via the Harvard Dataverse at https://doi.org/10.7910/DVN/FQJHKY 


\section{Appendix: Misinformation perceptions related to sources and issues across ten countries}

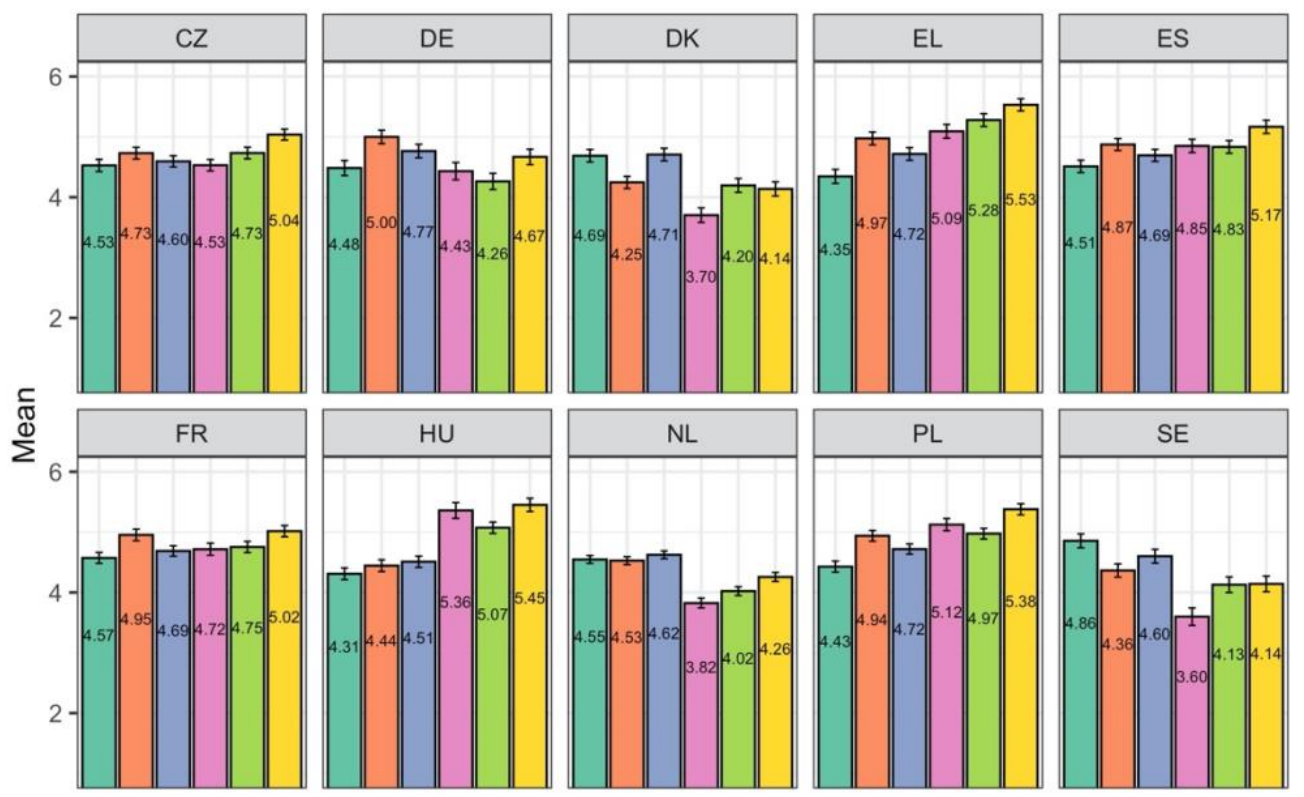

Sources of misinformation

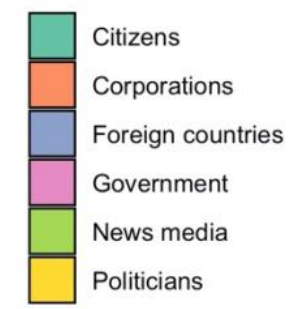

Figure A1. Perceived misinformation sources across ten countries.

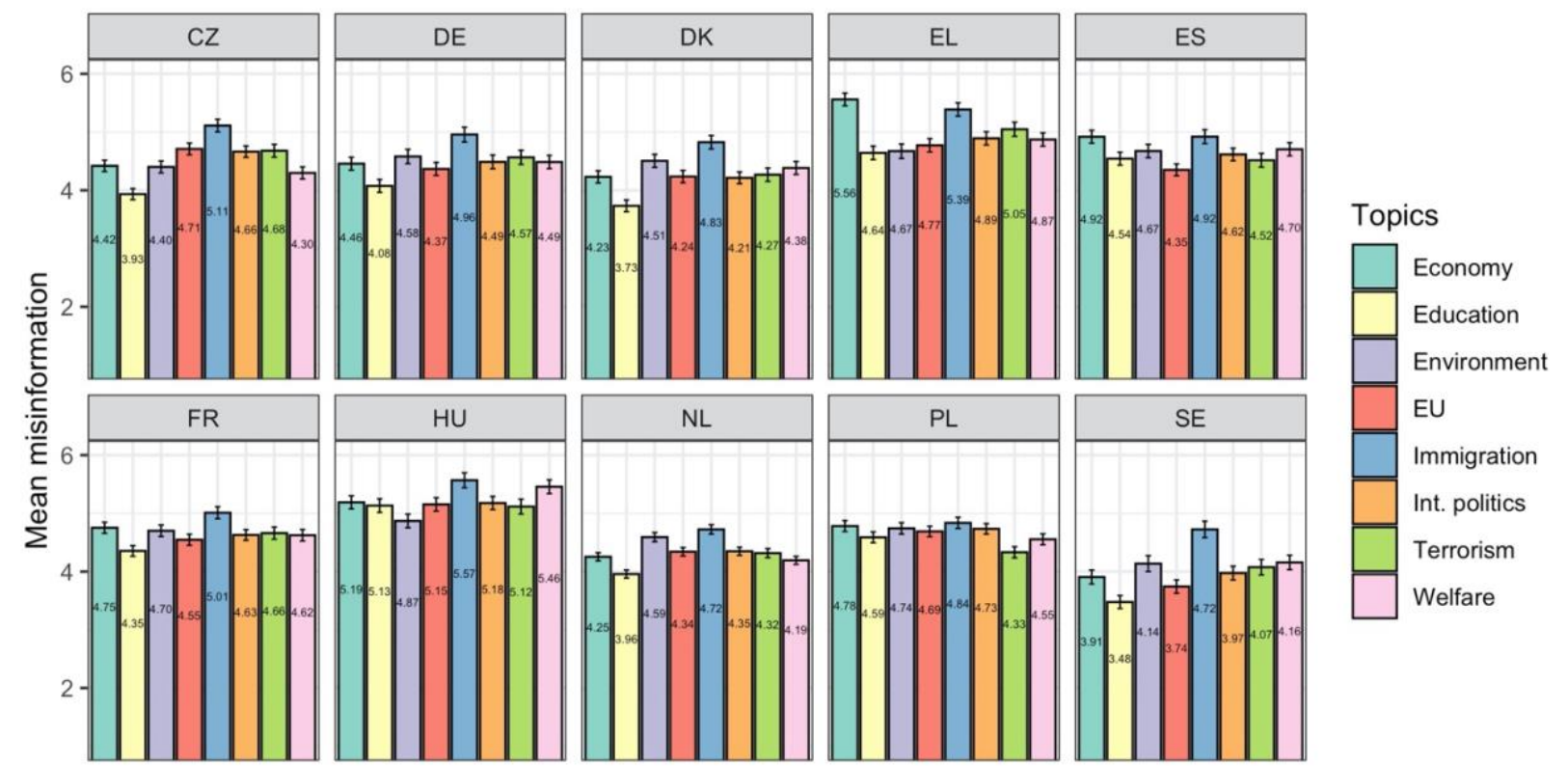

Figure A2. Perceived misinformation topics across ten countries 